FETAL AND NEONATAL EDITION

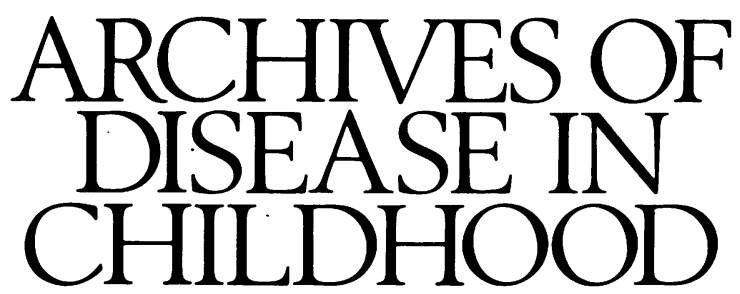

The fournal of the British Paediatric Association

\title{
Low cost technology for the newborn in developing countries
}

In 1924, the first paragraph of the Declaration of the Rights of the Child from a conference in Geneva, stated, 'the child must be given the means requisite for its normal development, both materially and spiritually'. ${ }^{1}$ Perinatology in developing countries has often failed to achieve this aim because of bureaucracy in local research grant giving bodies and also the lack of financial resources, planning, and appropriate teaching. The World Health Organisation (WHO) and the Commonwealth Association for Mental Handicap and Developmental Disabilities (CAMHADD) have both commented on the absence of basic facilities for resuscitation of the newborn with birth asphyxia, which is responsible for the death of more than one million babies per year and for an equal number of babies with hypoxic-ischaemic encephalopathy. ${ }^{2} 3$

Morley has said that perinatal care in developing countries could be improved more quickly than care in any other period. ${ }^{3}$ One of the answers is low cost technology for the newborn. The principle of this technology should be the same as that stressed by the Declaration of Alma Ata of WHO and Unicef in 1979: universal access at a cost that the community and country can afford. Too often the converse occurs - there is drainage of local capital to pay for sophisticated machines that often become unusable because of lack of maintenance; they are sometimes not even removed from their packaging.

There are a few reports of local initiatives to develop appropriate and alternative technology in developing countries, ${ }^{45}$ but they have been rarely evaluated objectively. ${ }^{6}$ One of these initiatives was described by Silva Fontes, ${ }^{5}$ a Brazilian 'do-it-yourself' neonatologist working in a public special care baby unit in Salvador, in the state of Bahia. He described more than 20 pieces of equipment designed and patented by himself at a fraction of the cost of buying standard equipment from medical suppliers. Among these was a pierced dummy adapted to accommodate a thermometer, a dropper (to introduce milk into the baby's mouth), or a suction catheter. According to him, it is particularly useful in babies with choanal atresia or Pierre Robin anomaly. Simple plastic food boxes can be transformed into headboxes for the supply of oxygen. He has also used other simple equipment: the opaque, plastic lid of the food box can serve as an eye shield during phototherapy; hand made wooden phototherapy units in which the baby lies in a hammock suspended between two rows of fluorescent lights; and low cost radiant warmers made of simple electric bulbs controlled by a dimmer switch. No incubators are used for the sickest babies, but cheap, ingenious, warmed open cots are used instead. All this equipment needs evaluation in collaboration with those in countries with more research resources.

Some researchers from industrialised countries have designed simple equipment that could be lifesaving for babies in the developing world. Because facilities for neonatal resuscitation are usually limited to major maternity units where only $10 \%$ of deliveries take place, simple apparatus is required. Milner et al described a simple effective method using a facemask resuscitation device. ${ }^{78}$ Inflation is provided by the operator blowing down a silicone tube containing a nylon wool filter to prevent cross infection. The other end of the silicone tube is attached to a round facemask. Positive pressure is provided by intermittent occlusion of a central hole in the mask. This device allows successful resuscitation at birth and gives a larger tidal volume than bag and mask systems. The maximum inspiratory pressures achieved by experienced and inexperienced volunteers were close. Both pressures and inspiratory times were similar to those generally applied for neonatal resuscitation. The price is below $\$ 5$ per unit. A device working on the same principle was reported by Morley in the 1970s, but could only be used with an endotracheal tube. ${ }^{3}$

Some collaborative research projects have been set up between the universities of developed and developing countries. For example there was an important study of the incidence of hypothermia in low birthweight infants admitted to a neonatal unit in Turkey, and the effect of a heated water filled mattress. ${ }^{6}$ The authors found that $35 \%$ of the infants had temperatures below $34^{\circ} \mathrm{C}$ on admission. Normal temperatures were achieved within the first day after admission in all infants treated on the heated water filled mattress whereas they were not achieved until three days later in the incubator group. This may be due to various factors: most neonatal units in developing 
countries are equipped with single wall incubators for economic reasons; the desired environmental temperature is not constant in air heated incubators because of fluctuating voltage in the main electrical supply. The warmth provided by the heated water filled mattress remains constant for long periods even if the electricity is cut off. It is interesting to note that the neonatal mortality was $21 \%$ with the mattress, compared with $34 \%$ in the incubator; however this did not achieve significance and a further study is required. This equipment, manufactured by Kanmed (Veddestavagen 13, S-17562 Jarfalla, Sweden) could be an ideal solution for developing countries. Although still quite expensive (approximately $\$ 1700$ per unit), similar equipment could probably be produced cheaper by a local university medical physics department, but it is essential that the safety standards should be the same.

By extension, the cheapest appropriate technology for a low birthweight newborn baby is close skin-to-skin contact with the mother. This method was shown by one of the authors to maintain the temperature of babies under $1500 \mathrm{~g}$ as effectively as in an incubator in the UK. ${ }^{9}$ This is one of the ideas behind the kangaroo mother method that is now being further evaluated. In a recent study conducted in Mozambique, 100 low birthweight infants surviving the first days of life (mean birth weight $1329 \mathrm{~g}$, mean gestational age 32.5 weeks, mean postnatal age 11.6 days), were nursed consecutively in the kangaroo mother section of the neonatal unit. ${ }^{10}$ They were all breast fed at discharge and the mean weight gain was $12.8 \mathrm{~g}$ per day. The neonatal mortality was $5 \%$ and the diarrhoea rate $8 \%$. Unfortunately this was not a randomised trial but nevertheless the late neonatal mortality rate in that nursery was usually about $18 \%$, and $15 \%$ suffered from diarrhoea. It was reported that babies were less likely to be abandoned in the hospital by their parents when this sort of intervention was used, which confirmed a previous report. ${ }^{11} \mathrm{~A}$ randomised controlled trial of very low birthweight babies showed that the duration of lactation of the mothers was significantly increased by around 4 weeks in the experimental group with skin-to-skin contact. ${ }^{9}$ This is clearly a major advantage in the developing world. The cultural acceptability of such methods of caring for babies may vary from country to country and will need to be better evaluated.

Much remains to be done to develop and assess scientifically appropriate low cost technology in developing countries. Paediatric units in industrialised countries should not only accept paediatricians for further training, but also a nurse and a biomedical maintenance technician and it would be better if they came as a team. Grant giving bodies should be encouraged to support research that would evaluate simple life saving techniques in the developing countries.

Paediatric Department,

D ACOLET

Oueen Charlotte's and Chelsea Hospital,

Goldhawk Road,

London W6 OX9

1 Fifth Committee to the Fifth Assembly, League of Nations. Declaration of the rights of the child. Declaration of Geneva. Geneva: League of Nations, 1924

2 Commonwealth Association for Mental Handicap and Developmental Disabilities. Report of the working group for development of a programme on prevention and management of birth asphyxia. CAMHADD Newsletter 1988; 8: 6-8.

3 Morley D. Paediatric priorities in the developing world. London: Butterworth, 1973.

4 Ebrahim GS. Care of the newborn in developing countries. London: Macmillan Press, 1979.

5 Silva Fontes JA. Unidade neonatal a baixo custo. in perinatologia social. São Paulo: Fundo Editorial BYK, 1984: 727-47.

6 Sarman I, Can G, Tunell R. Rewarming preterm infants on a heated, water filled mattress. Arch Dis Child 1989; 64: 687-92.

7 Hoskins EW, Milner AD, Hopkin IE. A simple method of face mask resuscitation at birth. Arch Dis Child 1987; 62: 376-8.

8 Milner AD, Upton CJ, Green J, Stokes GM. A device for domiciliary neonatal resuscitation. Lancet 1990 ; 335: 273-5.

9 Whitelaw A, Sleath K, Acolet D. Safety and effectiveness of skin-to-skin contact for very low birth weight infants. Pediatr Res 1988; 24: 269.

10 Colonna F, Uxa F, Da Graca AM, De Vonderweid U. The 'kangaroomother' method: evaluation of an alternative model for the care of low mother' method: evaluation of an alternative model for the care of low 31: 335-9.

11 Whitelaw A, Sleath K. Myth of the marsupial mother: home care of very low birth weight babies in Bogota, Colombia. Lancet 1985; i: 1206-8. 